\title{
Novel dihydrobenzofuro[4,5- $b][1,8]$ naphthyridin-6-one derivative, MHY-449, induces cell cycle arrest and apoptosis via the downregulation of Akt in human lung cancer cells
}

\author{
HYUN SOOK LIM ${ }^{1 *}$, YONG JUNG KANG ${ }^{1 *}$, BOKYUNG SUNG ${ }^{1}$, SEON HEE KIM ${ }^{1}$, \\ MIN JEONG KIM ${ }^{1}$, HYE RIM KIM ${ }^{1}$, SEONG JIN KIM ${ }^{1}$, YUNG HYUN CHOI ${ }^{2}$, \\ HYUNG RYONG MOON $^{1}$, HAE YOUNG CHUNG ${ }^{1}$ and NAM DEUK KIM ${ }^{1}$

\begin{abstract}
${ }^{1}$ College of Pharmacy, Molecular Inflammation Research Center for Aging Intervention (MRCA), Pusan National University, Busan 609-735; ${ }^{2}$ Department of Biochemistry, Dongeui University College of Oriental Medicine,
\end{abstract} \\ Busan 614-052, Republic of Korea
}

Received June 4, 2015; Accepted July 16, 2015

DOI: $10.3892 / o r .2015 .4208$

\begin{abstract}
The anticancer properties of MHY-449, a novel dihydrobenzofuro[4,5- $b][1,8]$ naphthyridin-6-one derivative, in various human cancer cell lines have been previously reported. The aim of the present study was to investigate the activities of MHY-449 on human lung cancer cells in order to elucidate its underlying molecular mechanisms of action. The result showed that MHY-449 treatment inhibited cell growth in a time- and concentration-dependent manner. Specifically, MHY-449 induced cell cycle arrest at the $S$ phase, and the resulting increased sub-G1 fraction led to the induction of apoptosis, as determined by flow cytometric analysis and DNA fragmentation. In addition, MHY-449 was shown to induce alterations in the ratio of $\mathrm{Bax} / \mathrm{Bcl}-2$ protein expression, and contribute to the loss of mitochondrial membrane potential. These cellular events then triggered the caspase cascade and subsequent poly(ADP-ribose) polymerase cleavage. The apoptotic cell death induced by MHY-449 was inhibited by pretreatment with Z-VAD-FMK, a pan-caspase inhibitor. Moreover, MHY-449 downregulated the phosphorylation of Akt, and the phosphatidylinositol-3 kinase/Akt inhibitor LY294002 was found to enhance its induction of apoptosis. Taken together, the results suggested that MHY-449 exerts anticancer effects by promoting cell cycle arrest and apoptosis via the downregulation of Akt. Based on these data, MHY-449
\end{abstract}

Correspondence to: Dr Nam Deuk Kim, Department of Pharmacy, College of Pharmacy, Pusan National University, Busandaehag-ro, 63 Beon-gil, Geumjeong-gu, Busan 609-735, Republic of Korea

E-mail: nadkim@pusan.ac.kr

*Contributed equally

Key words: MHY-449, lung cancer cells, cell cycle arrest, apoptosis, Akt serves as a potential candidate in the chemoprevention and/or treatment of lung cancer.

\section{Introduction}

Lung cancer is characterized by uncontrolled cell growth in tissues of the lung, trachea, or bronchi. This malignancy is currently the leading cause of cancer-related mortality in the world $(1,2)$. In Korea, the incidence of lung cancer increases gradually with age (3). Recently, Jung et al (1) reported that lung cancer is the fourth most prevalent type of malignancy, and the most common cause of cancer-related mortalities in Korea (1). Most $(\sim 85 \%)$ patients with lung cancer have the non-small cell lung cancer (NSCLC) subtype, and the majority of these patients have advanced disease (defined as stage IIIB or IV at the time of diagnosis) (4). The rapid developments in oncogene-directed targeted therapies have significantly changed the treatment paradigm of NSCLC during the last decade. However, tumors exposed to targeted therapies may develop acquired resistance against these treatments over time. Therefore, the development of novel agents to combat NSCLC, and associated resistance, is imperative.

The $( \pm)-\left(R^{*}\right)-5$-methoxy-11-methyl-2- $\left(\left(R^{*}\right)-2\right.$-methyloxiran2-yl)-1,2-dihydrobenzofuro[4,5- $b][1,8]$ naphthyridin-6(11H)-one, MHY-449, is a novel synthetic compound containing xanthone and acridone components, derived from psorospermin and acronycine templates, respectively (5). MHY-449 has been shown to induce apoptosis in various human cancer cell lines, including breast (5), colon (6) and prostate (5,7). While its precise mechanism of anticancer activity has not been fully elucidated, we have previously reported that MHY-449 induces G2/M phase cell cycle arrest $(5,6)$, inhibits Akt/forkhead box O1 (7), activates caspases (6) and extracellular signalregulated kinase (ERK) (7), modulates cell cycle regulatory proteins (6), and downregulates the anti-apoptotic protein Bcl-2 (6,7). These anticancer activities make MHY-449 an attractive candidate for potential pharmaceutical development. In this study, we evaluated the effect of MHY-449 on NSCLC cells in vitro. The results showed that MHY-449 exhibits a 
potent anticancer effect in NSCLC cells, attributable to its ability to induce apoptosis. It was also found that Akt is essential to MHY-449-induced apoptosis in these cells.

\section{Materials and methods}

Chemicals. The simplified code name and chemical structure of MHY-449 used in this study are shown in Fig. 1. The methods used for the design and synthesis of this compound have been previously described (ref?). MHY-449 was dissolved in dimethyl sulfoxide (DMSO) to yield a $10-\mathrm{mM}$ stock solution and stored at $-20^{\circ} \mathrm{C}$ until use. The maximal concentration of DMSO did not exceed $0.1 \%(\mathrm{v} / \mathrm{v})$ in the treatment range, where there was no influence on cell growth. DMSO and 3-(4,5-dimethylthiazol-2-yl)-2,5-diphenyl tetrazolium bromide (MTT) were obtained from Amresco LLC (Solon, OH, USA). Antibodies specific for pro-caspase-3, -8 , and -9 , poly(ADP-ribose) polymerases (PARP), BH3-interacting domain death agonist (Bid), Bcl-2-associated $\mathrm{X}$ protein (Bax), B-cell CLL/lymphoma 2 (Bcl-2), phospho-Akt, Akt, $\beta$-actin and Z-VAD-FMK, were obtained from Santa Cruz Biotechnology, Inc. (Dallas, TX, USA).

Cell culture and cell viability assay. The human A549 and NCI-H460 lung cancer cell lines were cultured at $37^{\circ} \mathrm{C}$ in humidified 5\% $\mathrm{CO}_{2}$ in RPMI-1640 medium supplemented with $10 \%$ fetal bovine serum (FBS), penicillin $(100 \mathrm{U} / \mathrm{ml})$, and streptomycin $(100 \mu \mathrm{g} / \mathrm{ml})$ (all from GE Healthcare Life Sciences, Logan, UT, USA). The non-transformed human Hs27 foreskin fibroblast cell line was cultured in Dulbecco's modified Eagle's medium (DMEM; GE Healthcare Life Sciences) supplemented with $10 \%$ FBS. To determine cell viability, the MTT assay was performed. The cells were seeded in 24-well culture plates, cultured for $24 \mathrm{~h}$, treated with or without various reagents at the indicated concentrations, and then incubated in the dark with MTT $(0.5 \mathrm{mg} / \mathrm{ml})$ at $37^{\circ} \mathrm{C}$ for $2 \mathrm{~h}$. The formazan granules generated by the live cells were dissolved in DMSO, and the absorbance at $540 \mathrm{~nm}$ was monitored using a multi-well reader (Thermo Fisher Scientific, Vantaa, Finland).

Annexin V/PI staining. To quantitatively determine the percentage of apoptotic cells, we used the BD Pharmingen FITC Annexin V Apoptosis Detection kit (BD Biosciences, San Diego, CA, USA) according to the manufacturer's instructions. The cells were stained with propidium iodide (PI) and Annexin V-fluorescein isothiocyanate (FITC) solution at room temperature for $15 \mathrm{~min}$ in the dark. The stained cells were then analyzed using flow cytometry within $1 \mathrm{~h}$.

DNA fragmentation assay. The cells were lysed in a buffer containing $5 \mathrm{mM}$ Tris- $\mathrm{HCl}$ ( $\mathrm{pH} 7.5$ ), $5 \mathrm{mM}$ ethylenediaminetetraacetic acid (EDTA), and $0.5 \%$ Triton X-100 for $30 \mathrm{~min}$ on ice. After centrifugation at $27,000 \mathrm{x}$ g for $15 \mathrm{~min}$, the fragmented DNA in the supernatant was treated with RNase, followed by proteinase $\mathrm{K}$ digestion, extraction with a phenol/chloroform/isoamyl alcohol mixture (25:24:1, v/v/v), and isopropanol precipitation. DNA was separated using a $1.6 \%$ agarose gel, stained with ethidium bromide $(0.1 \mu \mathrm{g} / \mathrm{ml})$, and visualized using an ultraviolet source.

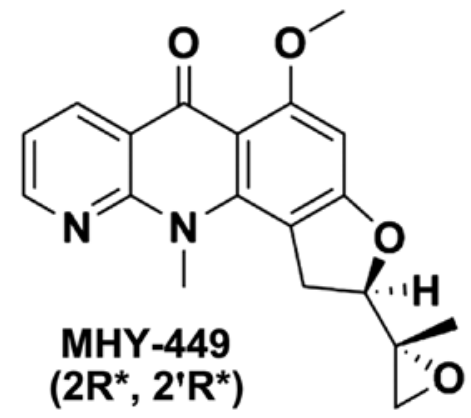

Figure 1. The chemical structure of MHY-449 $\left[( \pm)-\left(R^{*}\right)-5-\right.$ methoxy-11-methyl-2- $\left.R^{*}\right)$-2-methyloxiran-2-yl)-1,2-dihydrobenzofuro[4,5- $b$ ] $[1,8]$ naphthyridin-6(11H)-one]

Western blot analysis. The cells were harvested, lysed, and equal amounts of protein were subjected to sodium dodecyl sulfate-polyacrylamide gel electrophoresis (SDS-PAGE) and transferred to polyvinylidene fluoride (PVDF) membranes for immunoblotting. Blots were probed with the desired primary antibodies overnight, incubated with horseradish peroxidase (HRP)-conjugated secondary antibodies (Santa Cruz Biotechnology, Inc.), and then visualized using an enhanced chemiluminescence (ECL) detection system (GE Healthcare, Piscataway, NJ, USA).

Measurement of mitochondrial membrane potential (MMP, $\Delta \Psi m)$. MMP was determined using a flow cytometer and the lipophilic cationic dye 5,5',6,6'-tetrachloro-1,1',3,3'-tetra-ethylbenzimidazolylcarbocyanine iodide (JC-1; Calbiochem, San Diego, CA, USA). JC- 1 is a dye that stains the mitochondria of living cells in a membrane potential-dependent manner. The cells were treated with various concentrations of JC-1, harvested, and washed with cold PBS. The cells were stained with $10 \mu \mathrm{M}$ JC-1 for $20 \mathrm{~min}$ at $37^{\circ} \mathrm{C}$ in the dark. The cells were subsequently washed with cold PBS and then analyzed using an Accuri C6 flow cytometer (BD Biosciences, Ann Arbor, MI, USA).

Flow cytometric analysis of sub-G1 phase and cell cycle population. The DNA content was measured following the staining of the cells with PI (Sigma-Aldrich Co., LLC, St. Louis, MO, USA). After treatment with various concentrations of MHY-449, the cells were collected, washed with cold PBS, and then fixed in $70 \%$ ethanol at $-20^{\circ} \mathrm{C}$ overnight. The fixed cells were washed with cold PBS and then stained with cold PI solution $\left(50 \mu \mathrm{g} / \mathrm{ml}\right.$ in PBS) at $37^{\circ} \mathrm{C}$ for $30 \mathrm{~min}$ in the dark. Analysis was performed using an Accuri $\mathrm{C} 6$ flow cytometer.

Statistical analysis. Results were presented as the mean \pm standard deviation (SD) of three separate experiments and analyzed using the Student's t-test. The acceptable level of significance was established at $\mathrm{P}<0.05$.

\section{Results}

MHY-449 inhibits the proliferation of lung cancer cells. The anti-proliferative activities of MHY-449 on NSCLC cell lines A549 and NCI-H460, were first evaluated using the MTT cell viability assay. As shown in Fig. 2, MHY-449 reduced cell viability in a concentration- and time-dependent manner in 
each of the cell lines. The A549 cell line was more sensitive to the effects of MHY-449 in comparison to the NCI-H460 cell line. The $\mathrm{IC}_{50}$ of MHY-449 was found to be $0.66 \mu \mathrm{M}$ for A549 cells (Fig. 2A) and $1.57 \mu \mathrm{M}$ for NCI-H460 cells (Fig. 2B) at $48 \mathrm{~h}$. In subsequent experiments, we used MHY-449 at concentrations up to 1 and $2 \mu \mathrm{M}$ for use with A549 and NCI-H460 cells, respectively.

The effects of MHY-449 on the normal Hs27 cell line were also analyzed (Fig. 2C). MHY-449 (2 $\mu \mathrm{M})$ inhibited $>40 \%$ of the cell growth in A549 and NCI-H460 lung cancer cell lines, whereas little growth inhibition was oberved in the non-transformed human Hs27 foreskin fibroblast cell line.

MHY-449 induces apoptotic cell population in NSCLC cells. To examine the mechanisms responsible for MHY-449-meditated cell growth inhibition, its effect on apoptosis induction was examined in NSCLC cells. The A549 and NCI-H460 NSCLC cell lines were treated with different concentrations of MHY-449 for $24 \mathrm{~h}$. An increased proportion of A549 cells was found to be in the sub-G1 phase (29.6\%) $24 \mathrm{~h}$ after treatment with MHY-449 $(1 \mu \mathrm{M})$ compared to the vehicle-treated control cells $(1.7 \%)$. However, the ability of MHY-449 to promote apoptosis in NCI-H460 cells was modest compared to this effect in A549 cells. As shown in Fig. 3, MHY-449 only slightly altered the percentage of NCI-H460 cells in the sub-G1 phase. Specifically, MHY-449 $(2 \mu \mathrm{M})$ treatment increased the sub-G1 phase cell population from $2.4 \%$ (vehicle-treated control) to $10 \%$. These results suggested that MHY-449 induces apoptosis in NSCLC cells in a concentration-dependent manner.

MHY-449 treatment induces apoptosis in NSCLC cells. To confirm that the effects of MHY-449 in NSCLC cells are associated with the induction of apoptosis, we performed flow cytometric analyses using cells stained with Annexin V/PI. Exposure of A549 and NCI-H460 cells to increasing concentrations of MHY-449 for $24 \mathrm{~h}$ resulted in a gradual increase in the apoptotic cell population. Fig. 4A shows that MHY-449 increased the proportions of early (lower-right quadrant) and late (upper-right quadrant) apoptotic cells with increasing concentration in the two cell lines. For example, $33.9 \%$ of cells advanced to late apoptosis-induced death following treatment with MHY-449 $(1 \mu \mathrm{M})$ as compared to the number of apoptotic cells in the vehicle-treated control (4.5\%).

We determined the effect of MHY-449 on DNA fragmentation in A549 and NCI-H460 cells, another hallmark event of apoptosis. As shown in Fig. 4B, the ladder pattern of DNA was observed in the NSCLC cells in a concentration-dependent manner with respect to MHY-449. In A549 cells, this typical ladder pattern of internucleosomal fragmentation resulted from exposure to $1 \mu \mathrm{M}$ MHY-449, whereas in NCI-H460 cells, $2 \mu \mathrm{M}$ concentrations were required (Fig. 4B).

These results indicated that MHY-449 induces apoptosis in lung cancer cells. To determine the possible origin of this effect, we investigated the influence of MHY-449 on apoptosis-regulated gene expression. In particular, we assessed the potential of MHY-449 to alter the expression levels of caspases in NSCLC cells. We found that treating cells with MHY-449 resulted in a concentration-dependent decrease of pro-caspase-8, -9 , and -3 levels, and also cleavage of their substrate PARP (Fig. 4C).
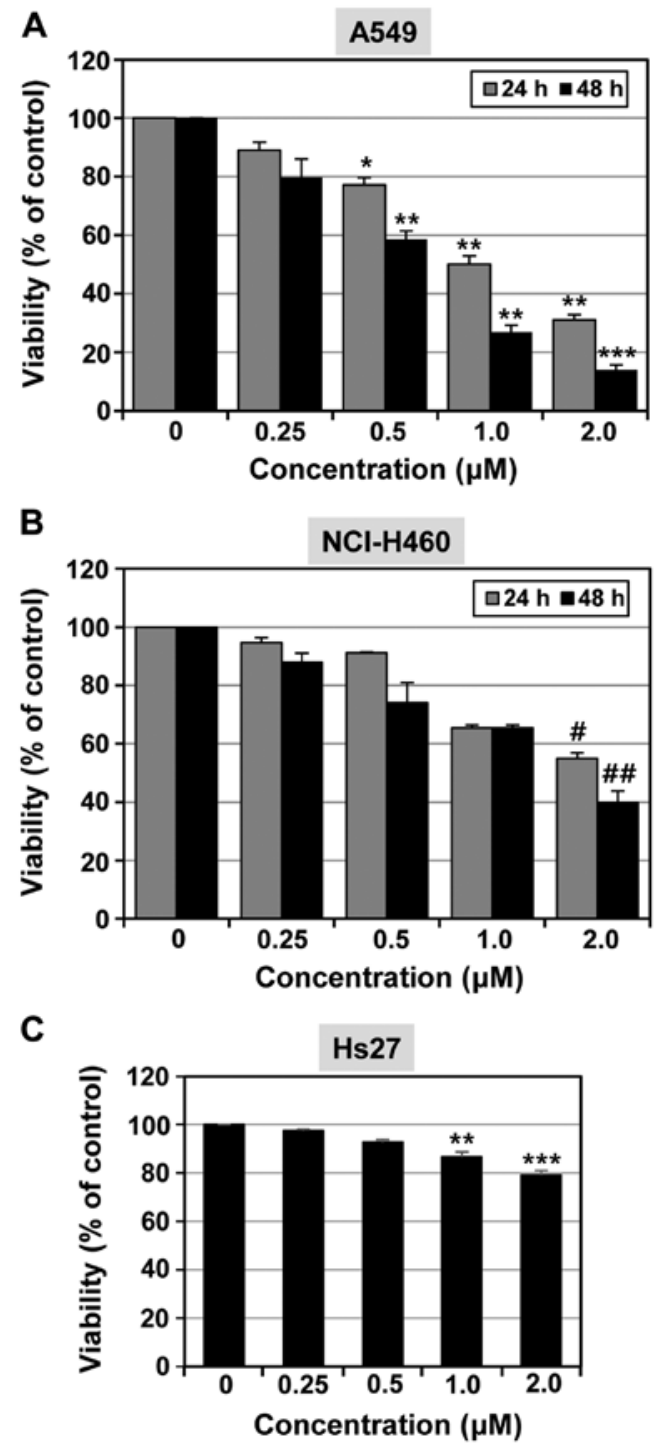

Figure 2. The effect of MHY-449 on the viability of human A549 and NCI-H460 lung cancer cell lines. (A and B) The cells were incubated with increasing concentrations of MHY-449 for 24 and $48 \mathrm{~h}$, and then the percentage of cell survival was determined using the MTT assay. (C) Hs27 cells were treated with MHY-449 for $24 \mathrm{~h}$, and the percentage of cell survival was determined by the MTT assay. Results were expressed as a percentage of vehicle treated control $\pm \mathrm{SD}$ of three separate experiments. The significance was determined using the Student's t-test $\left({ }^{*} \mathrm{P}<0.05,{ }^{* *} \mathrm{P}<0.01\right.$ and ${ }^{* * *} \mathrm{P}<0.001$ vs. vehicle-treated cells; ${ }^{\#} \mathrm{P}<0.05$ and ${ }^{\# \#} \mathrm{P}<0.01$ vs. vehicle-treated cells.

As mitochondria play a critical role in apoptosis triggered by a variety of stimuli (8), we investigated the effect of MHY-449 on signaling molecules known to be involved in these pathways. Exposure of NSCLC cells to MHY-449 triggered a downregulation of the whole form of pro-apoptotic protein Bid, resulting from Bid cleavage and activation. MHY-449 was also found to increase the levels of the pro-apoptotic protein Bax in A549 cells. No analogous alterations of Bax expression were observed in NCI-H460 cells because of MHY-449 treatment. A clear decrease in (anti-apoptotic protein) Bcl-2 expression was observed in the two NSCLC cell lines (Fig. 4C) following treatment with MHY-449. These results suggested that, MHY-449 induces the apoptosis of human lung cancer cells via activation of the caspase cascade and through the mitochondrial pathway. 

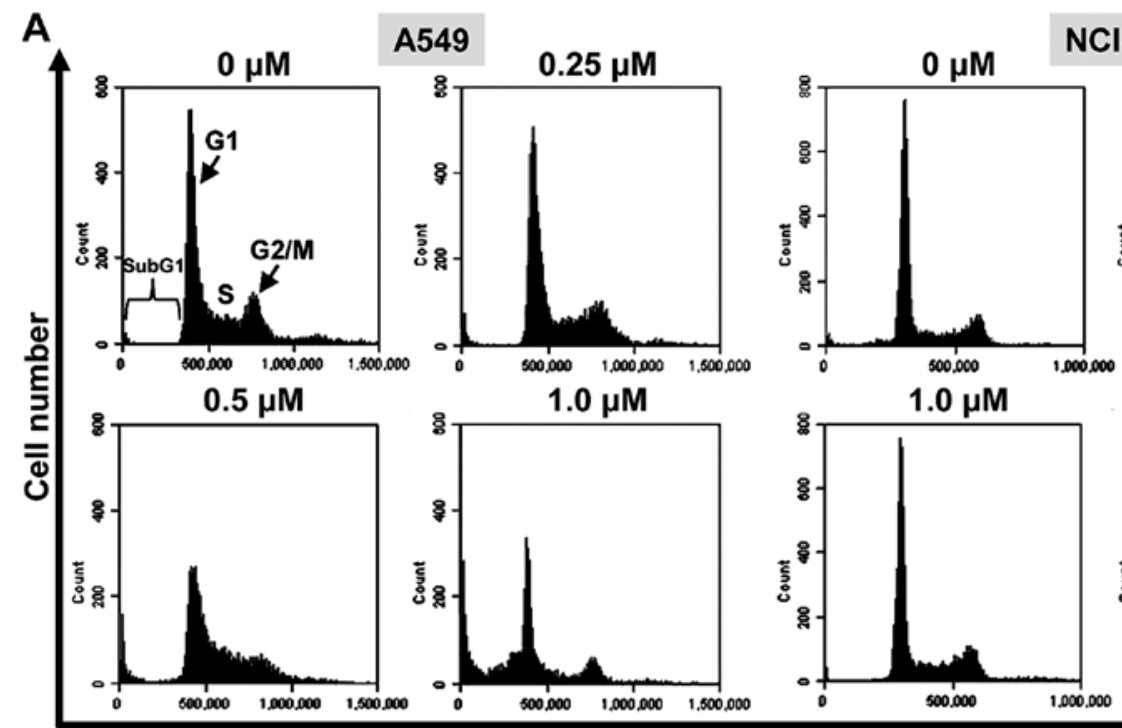

$\mathrm{NCl}-\mathrm{H} 460$
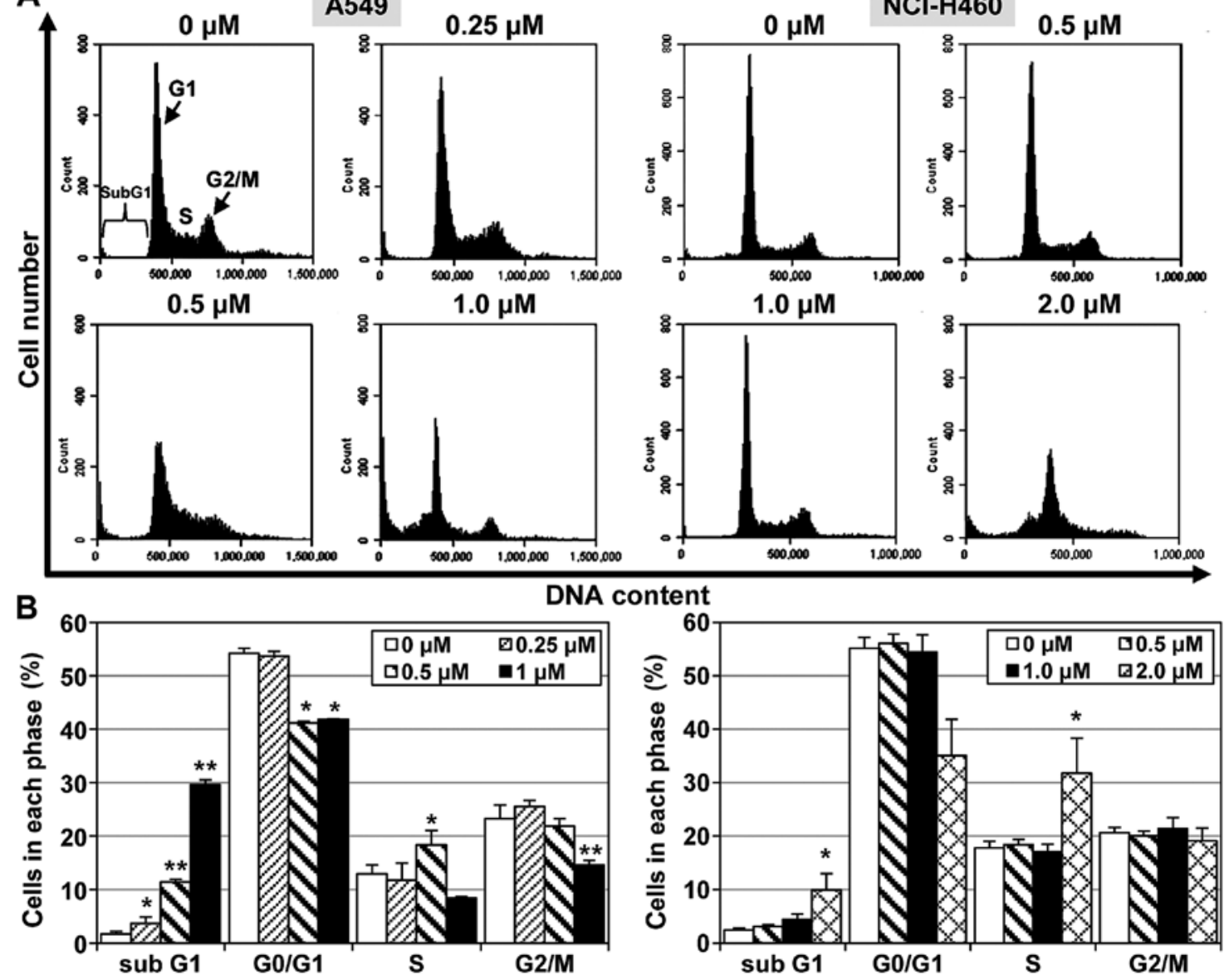

Figure 3. The effect of MHY-449 on cell cycle distribution in A549 and NCI-H460 cells. (A) Cells were treated with the indicated concentrations of MHY-449 for $24 \mathrm{~h}$, stained with PI, and then subjected to flow cytometric analysis to determine their distribution in each phase of the cell cycle. The representative results from three independent experiments are shown. (B) Results are shown as the mean \pm SD of four independent experiments. The significance was determined using the Student's t-test ( $\mathrm{P}<0.05$, and ${ }^{* *} \mathrm{P}<0.01$ vs. vehicle-treated cells). PI, propidium iodide.

Caspases are involved in MHY-449-induced apoptosis in NSCLC cells. To investigate the significance of caspase activation in MHY-449-induced apoptosis, we determined the viability of NSCLC cells pretreated with Z-VAD-FMK, a broad-spectrum caspase inhibitor, for $1 \mathrm{~h}$ followed by treatment with MHY-449 for $24 \mathrm{~h}$. As shown in Fig. 4D, pretreatment of A549 cells with Z-VAD-FMK promoted their viability as compared to cells treated with MHY-449 only. Similar results were obtained using NCI-H460 cells (Fig. 4D). To verify the role of caspase activation in MHY-449-induced apoptosis, we determined apoptotic cell death by analyzing the progressive proteolytic cleavage product of PARP, an activated caspase-3 substrate protein. Pretreatment of the cells with Z-VAD-FMK was found to abolish MHY-449-induced cleavage of PARP (Fig. 4E). These results suggested that activation of the caspase cascade is involved in the induction of apoptosis by MHY-449.

MHY-449 promotes loss of the mitochondrial membrane potential. The alteration of Bax, Bid, and Bcl-2 proteins are thought to contribute to apoptotic cell death by promoting the release of apoptogenic molecules from the mitochondria into the cytosol. As the expression levels of Bax, Bid, and Bcl-2 in NSCLC cells were found to be influenced by MHY-449 (Fig. 4C), we determined whether the resulting death of these cells was associated with disruption of the mitochondrial membrane potential (MMP). The effect of MHY-449 on MMP was determined by flow cytometric analyses following staining of the examined cells with JC-1, a lipophilic cationic dye that selectively enters mitochondria. As shown in Fig. 5A and B, JC-1 in the control cells exhibited red fluorescence due to the accumulation of J-aggregates indicating intact MMP (upper panel). By contrast, treating the cells with MHY-449 resulted in a concentration-dependent green fluorescence (lower panel) resulting from the cytosolic accumulation of monomeric JC-1. The populations of A549 cells with a disrupted membrane potential were 20.2, 29.2, 41.2 and $63.7 \%$ at $0,0.25,0.5$, and $1 \mu \mathrm{M}$ MHY-449 concentrations, respectively (Fig. 5C). MHY-449 $(1 \mu \mathrm{M})$ also increased the population of NCI-H460 cells with a disrupted membrane potential to $41.6 \%$ compared to that observed in the control (17\%; Fig. 5C). Collectively, these results indicated that MHY-449 induces a loss of MMP in NSCLC cells.

Akt inhibitor enhances apoptosis induced by MHY-449. Recent evidence has determined that Akt signaling is essential for NSCLC cell survival (9). Activation of the Akt pathway renders cells resistant to apoptosis via the regulation of proand anti-apoptotic proteins $(10,11)$. It was also reported that inhibition of the Akt pathway served to arrest cancer cell 
A

A $\quad 0 \mu \mathrm{M} \quad$ A549

$0.25 \mu \mathrm{M}$
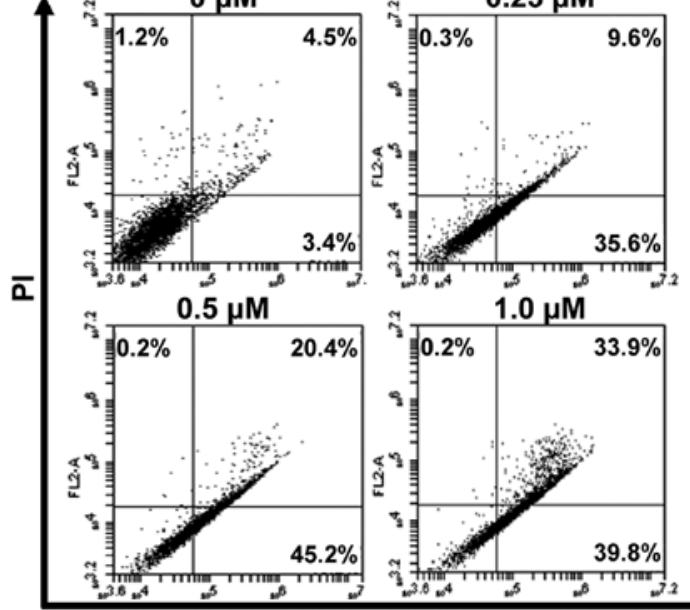

$35.6 \%$
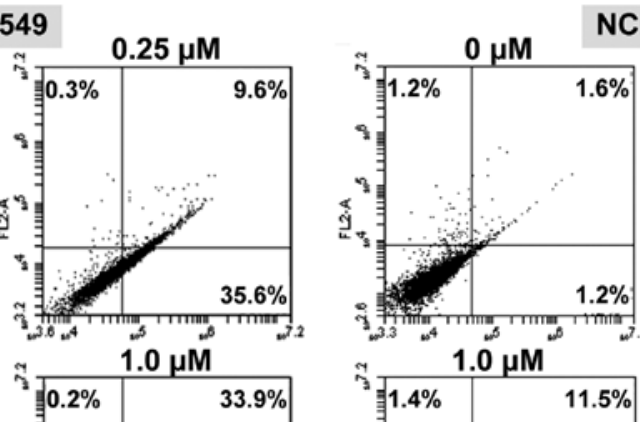

$\mathrm{NCl}-\mathrm{H} 460$
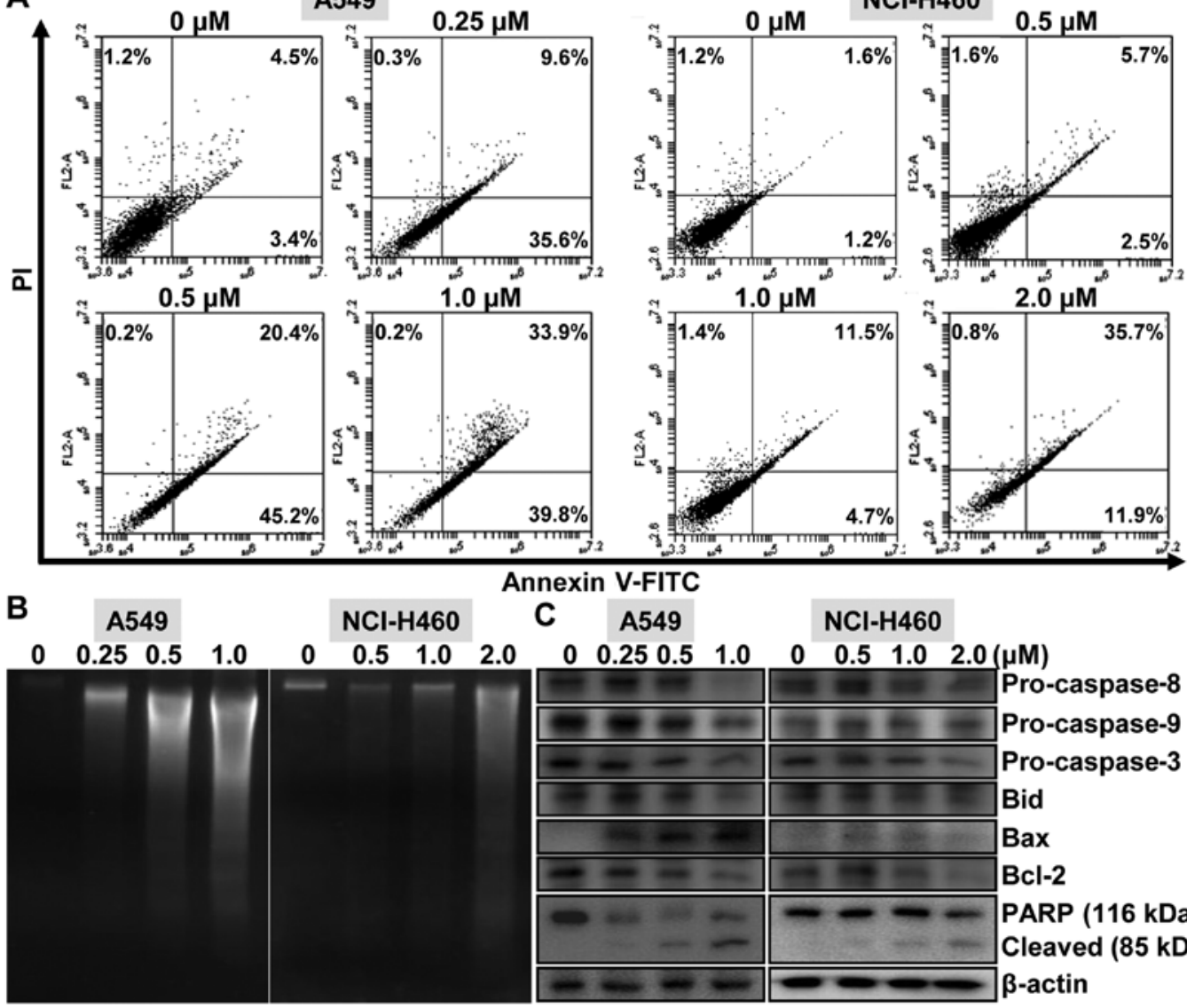

$39.8 \%$
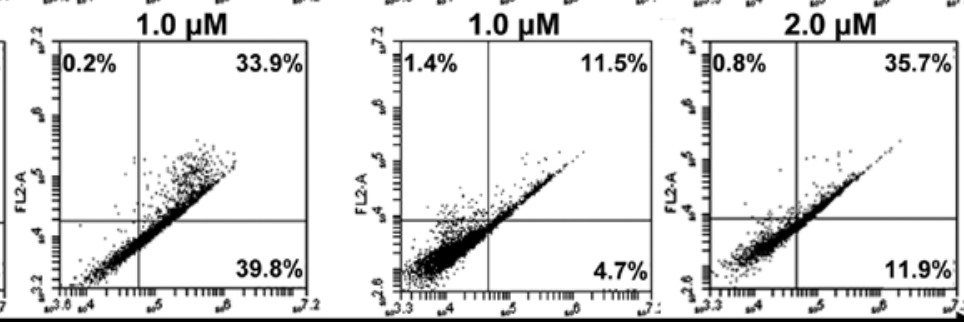

Annexin V-FITC

C A549

$\mathrm{NCl}-\mathrm{H} 460$

$\begin{array}{llllllll}0 & 0.25 & 0.5 & 1.0 & 0 & 0.5 & 1.0 & 2.0\end{array}$

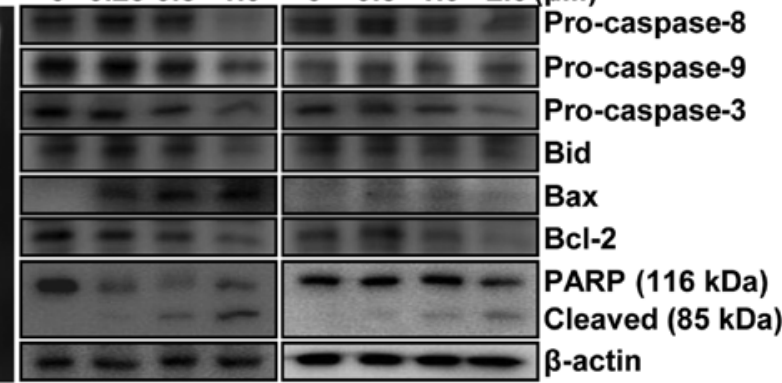

D

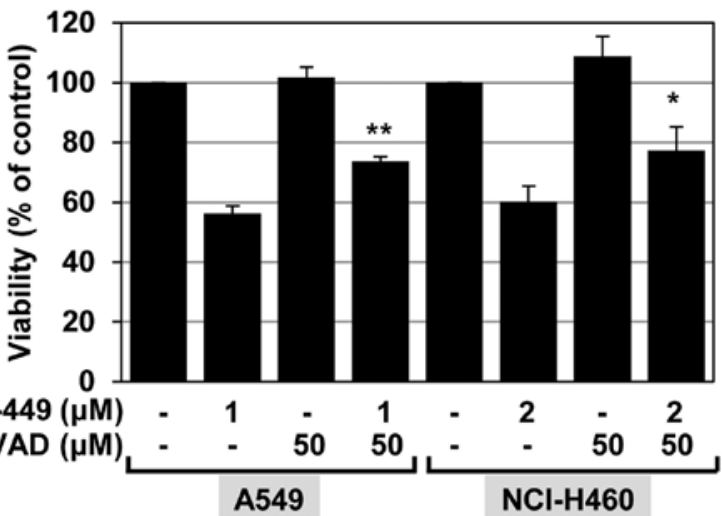

E

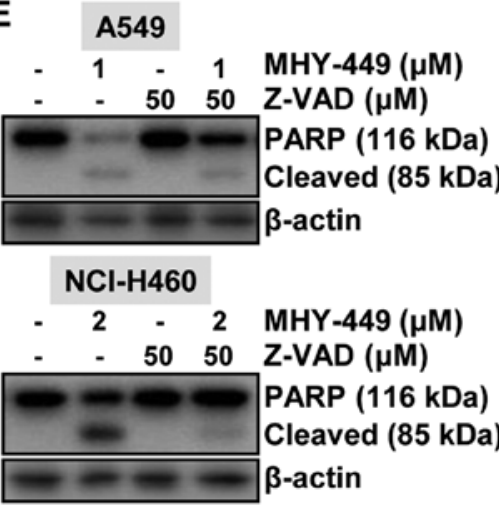

Figure 4. The effect of MHY-449 on the induction of apoptosis in A549 and NCI-H460 cells. (A) To investigate the effect of MHY-449 on cell death, the cells were treated for $24 \mathrm{~h}$ with the indicated concentrations of MHY-449. The cells were stained with Annexin V-FITC/PI and analyzed by flow cytometry. (B) To analyze the fragmentation of genomic DNA, the DNA was extracted from the cells and detected by $1.6 \%$ agarose gel electrophoresis in the presence of EtBr. (C) The expression levels of proteins involved in the extrinsic and intrinsic apoptotic pathways, such as pro-caspase- 8 , -9 and -3 , Bid, Bax, Bcl-2 and PARP $(116 \mathrm{kDa})$, were detected by western blot analysis. Proteins were visualized using the ECL detection system. $\beta$-actin was used as an internal control. (D) Cells were treated with the indicated concentrations of MHY-449 for $24 \mathrm{~h}$ after pretreatment with Z-VAD-FMK $(50 \mu \mathrm{M})$ for $1 \mathrm{~h}$. The degree of cytotoxicity was determined using the MTT assay. Data are shown as mean \pm SD of three independent experiments ${ }^{* * *} \mathrm{P}<0.01$ vs. MHY- 449 -treated A549 cells and ${ }^{*} \mathrm{P}<0.05$ vs. MHY-449-treated NCI-H460 cells). (E) Cells were pretreated with Z-VAD-FMK $(50 \mu \mathrm{M})$ for $1 \mathrm{~h}$ then exposed to MHY-449 for $20 \mathrm{~h}$. Total cell lysates were prepared and analyzed by western blot analysis. $\beta$-actin was used as a protein loading control. The representative results from three independent experiments are shown. FITC, fluorescein isothiocyanate; PI, propidium iodide; Bid, BH3-interacting domain death agonist; Bax, Bcl-2-associated X protein; Bcl-2, B-cell CLL/lymphoma 2; PARP, poly(ADP-ribose) polymerase.

proliferation and significantly delay tumor growth $(12,13)$. Thus, we examined the involvement of Akt signaling in mediating apoptosis induced by MHY-449. Treatment of A549 cells with the Akt inhibitor LY294002 alone had a minimal effect on inducing apoptosis (Fig. 6A). We also observed no notable induction of apoptosis in NCI-H460 cells treated with LY294002 (Fig. 6A). We also determined the influence of Akt inhibition on MHY-449-induced apoptosis in NSCLC cells. As shown in Fig. 6A, LY294002 enhanced the proportion of cells that underwent MHY-449-induced apoptosis in the NSCLC cell lines evaluated. Specifically, this proportion increased from 18.1 to $35.9 \%$ in A549 cells and from 9.8 to $21.6 \%$ in NCI-H460 cells. These results indicated that Akt inhibition enhances the apoptotic effect of MHY-449 in NSCLC cells. 

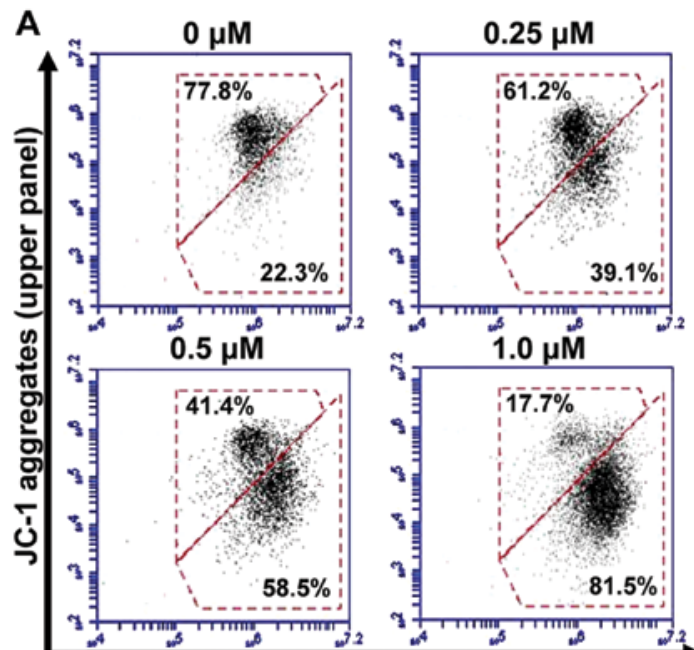

JC-1 monomers (lower panel)

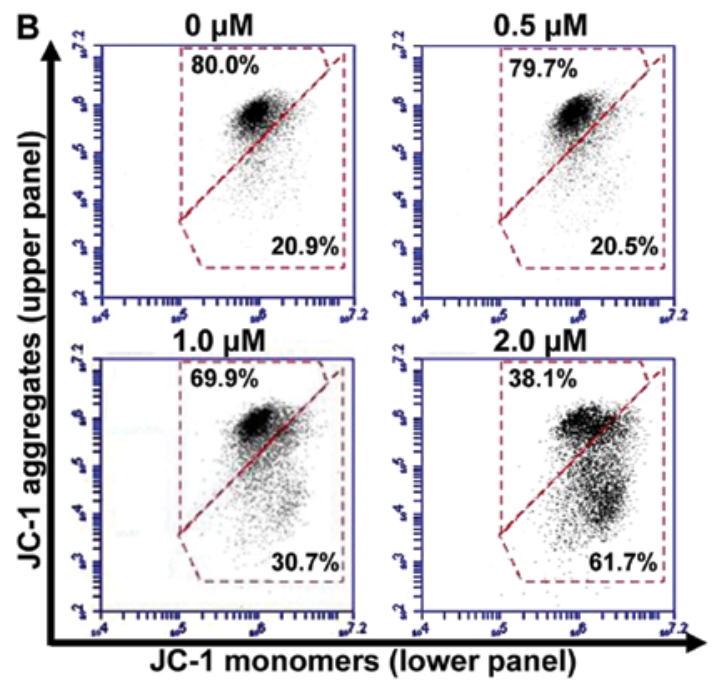

C

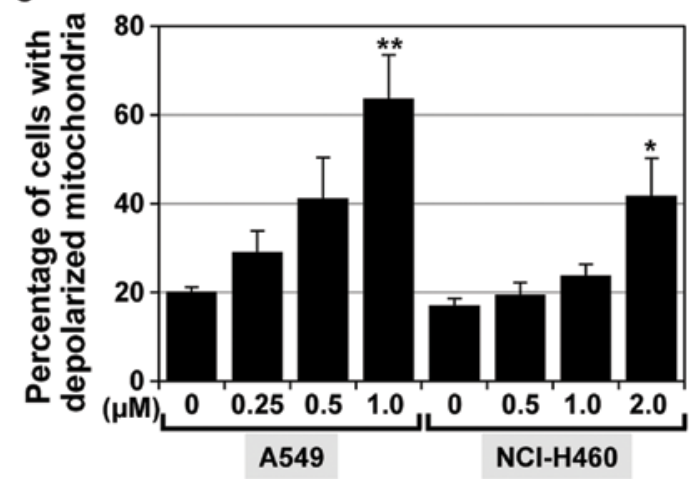

Figure 5. The effect of MHY-449 on mitochondrial membrane potential $(\Delta \Psi \mathrm{m})$ in A549 and NCI-H460 cells. To analyze $\Delta \Psi \mathrm{m}$, (A) A549 cells and (B) NCI-H460 cells were incubated with the indicated concentrations of MHY-449 for $24 \mathrm{~h}$, stained with JC-1 dye, and analyzed using flow cytometry. The data shown are representative of three independent experiments with similar results. (C) The quantitative data are presented as means of green fluorescence (depolarized $\Delta \Psi \mathrm{m}$ ) from the monomeric form of JC-1. Results are shown as the mean \pm SD of three separate experiments. The significance was determined using the Student's t-test $\left({ }^{*} \mathrm{P}<0.05\right.$ and ${ }^{* *} \mathrm{P}<0.01$ vs. vehicle-treated cells).

To confirm this result, we performed western blot analysis to investigate the effect of LY294002, MHY-449, and their co-administration on Akt activation and PARP cleavage.
A

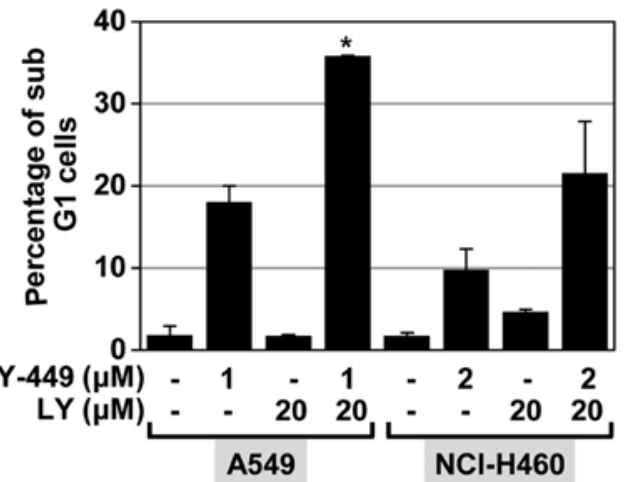

B

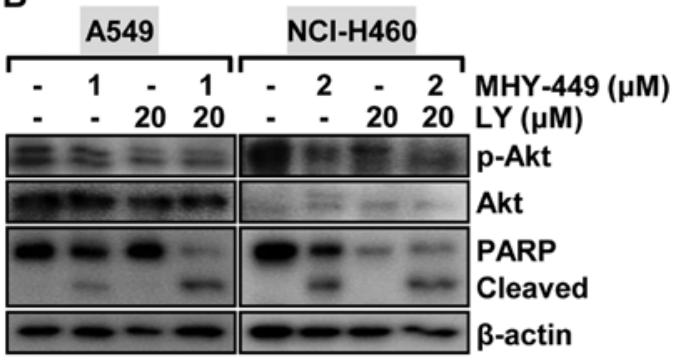

Figure 6. The role of Akt on MHY-449-induced apoptosis in A549 and NCI-H460 cells. (A) Cells were incubated with the indicated concentrations of MHY-449 for $20 \mathrm{~h}$ after pretreatment with LY294002 $(20 \mu \mathrm{M})$ for $1 \mathrm{~h}$. Cells were analyzed by flow cytometry following staining with PI. The presence of cells with sub-G1 DNA content following treatment with MHY449 was evaluated using flow cytometry in order to quantify the onset of apoptosis. Results are shown as the mean \pm SD of three separate experiments. The significance was determined using the Student's t-test ( $\mathrm{P}<0.05$ vs. MHY-449-treated cells). (B) Cells were pretreated with LY294002 $(20 \mu \mathrm{M})$ for $1 \mathrm{~h}$ and then exposed to MHY-449 for $24 \mathrm{~h}$. Total cell lysates were prepared and immunoblotted using p-Akt (Ser473), Akt, PARP antibodies and ECL detection. $\beta$-actin was used as a protein loading control. The representative results from three independent experiments are shown. PI, propidium iodide; PARP, poly(ADP-ribose) polymerase.

LY294002 was found to inhibit Akt activation in the two lung cancer cell lines evaluated. Of note, the concentrations of phosphorylated Akt (active form) were markedly reduced after these cells were treated with MHY-449 (Fig. 6B). Treatment of the NSCLC cells with LY294002 and MHY-449 was found to significantly suppress Akt activation (Fig. 6B). As such, the apoptogenic effect of MHY-449 on NSCLC cells was markedly enhanced by LY294002 (Fig. 6B). These results suggested that the Akt pathway is likely involved in MHY-449-induced apoptosis of NSCLC cells.

\section{Discussion}

In the present study, we have determined that synthetic MHY-449 exerts potent antitumor effects in NSCLC cells. To the best of our knowledge, this is the first study describing this effect of MHY-449 on NSCLC cells, despite its documented cytotoxicity in numerous other cancer cell lines (5-7). MHY-449 can effectively induce NSCLC cells to undergo apoptosis. When the underlying mechanism for this effect was examined, we found that MHY-449 triggered the caspase cascade, and also induced apoptosis through the mitochondrial pathway. Therefore, a resulting loss of mitochondrial membrane potential is likely a primary mechanism by which MHY-449 exerts antitumor effects in NSCLC cells. 
We also demonstrated that concentrations of phosphorylated Akt were markedly reduced in MHY-449-treated cells. Furthermore, the pharmacologic inhibition of Akt was found to markedly enhance MHY-449-induced NSCLC cell apoptosis. Constitutive activation of the Akt pathway is frequently observed in NSCLC cells, which acts to sustain their proliferation and survival. MHY-449 may be considered in the treatment of lung cancer owing to its apoptogenic properties in these cells, and ability to effectively suppress the Akt pathway.

Apoptosis is an important event leading to programmed cell death, which is often essential for the physiologic development and maintenance of organisms (14). The present study demonstrates that MHY-449 exerts a pronounced cytotoxic effect in two different NSCLC cell lines. MHY-449 was shown to effectively decrease the proliferation of A549 cells, which were more sensitive to this agent than NCI-H460 cells. MHY-449 also produced clear increases in sub-G1 populations and DNA fragmentation patterns in these cell lines. Phosphatidylserine exposure on the external plasma membrane leaflet in MHY-449-treated NSCLC cells was confirmed by Annexin $\mathrm{V}$ staining. Collectively, these results suggest that MHY-449 effectively inhibits NSCLC cell proliferation and induces apoptosis in a concentration-dependent manner.

The caspase family of cysteine proteases plays a central role in regulating apoptosis. It is has been well-established that certain caspases (e.g., caspase-8 and -9) play upstream initiator roles in apoptosis by coupling cell death to stimuli from the downstream effector caspases (e.g., caspase-3, the most significant promotor of apoptosis) (15). Of note, the downregulation of caspases-3, -9 , and -8 , as well as cleavage of PARP, was observed in present study. The observed caspase-mediated properties of MHY-449 are in agreement with previous studies using colon (6) and prostate (7) cancer cells. We also demonstrated that MHY-449, in the presence of Z-VAD-FMK, prevented the degradation of PARP in these cells. However, Z-VAD-FMK did not effectively rescue NSCLC cells from MHY-449-induced apoptosis. Thus, these results suggest that MHY-449-triggered apoptosis is likely mediated, at least in part, by the caspase cascade.

Since mitochondria play a crucial role in the extrinsic and intrinsic pathways of apoptosis (16), we also examined the effect of MHY-449 on mitochondrial function. Members of hte Bcl-2 family of proteins are known to govern apoptotic cell death either as activators (e.g., Bad, Bax, and Bid) or inhibitors (e.g., Bcl-2, Bcl-xL, and Bcl-W) of mitochondrial outer membrane permeabilization (16). For example, Bid is readily cleaved by caspase- 8 following stimulation of the extrinsic apoptotic pathway (17). Our results indicate that the expression of full-length Bid decreased following exposure to MHY-449, which then led to its cleavage. The results also demonstrated that MHY-449 served to simultaneously decrease $\mathrm{Bcl}-2$ expression and increase Bax expression. This resulting decrease in the $\mathrm{Bcl}-2 / \mathrm{Bax}$ ratio is known to be a critical factor in determining which cells undergo apoptosis (18). In addition, the level of MMP disruption was enhanced by MHY-449 in NSCLC cells. Taken together, these results suggest that MHY-449-induced apoptosis is associated with alteration of the Bcl-2/Bax ratio and downregulation of the full-length Bid, which collectively induce outer mitochondrial membrane permeabilization and the loss of MMP.
We observed that MHY-449 represses phosphorylated Akt expression in NSCLC cells. In agreement with this finding, Akt activation has been shown to be negatively regulated by MHY-449 in human prostate cancer cells (7). In addition, we demonstrated that exposing NSCLC cells to an Akt inhibitor (LY294002, $20 \mu \mathrm{M}$ ) alone only minimally induces cell death. However, concomitant treatment of cells with LY294002 and MHY-449 resulted in enhanced apoptotic cell death. Given the established role of the phosphatidylinositol-3 kinase/ Akt/mammalian target of rapamycin (PI3K/Akt/mTOR) signaling cascade as a pivotal pathway in cancer cell growth and survival (19-21), an agent that can perturb this signaling sequence may serve to prevent and/or treat tumor progression. Previous studies have indicated that NSCLC cells highly express phosphorylated and activated Akt $(22,23)$. Additionally, the tumor-suppressor gene phosphatase and tensin homolog (PTEN) is involved in the regulation of cell survival as well as apoptosis through the PI3K/Akt pathway. Specifically, the downregulation of PTEN is accompanied by a corresponding upregulation of phospho-Akt levels (19). We found that A549 cells were more sensitive to the combination of LY294002 and MHY-449 than were NCI-H460 cells. The phosphorylation of Akt is known to occur more readily in A549 cells than in NCI-H460 cells, since A549 cells harbor more methylated promoter CpG sites in PTEN $(11,24)$. Although we have yet to determine the precise effect(s) of MHY-449 on PTEN, the known discrepancy in Akt phosphorylation between A549 and H460 cells may explain their difference in susceptibility to concomitant LY294002/MHY-449 treatment.

In conclusion, the results of the present study have provided evidence that MHY-449 induces human NSCLC cell death stemming from activation of the caspase family and PARP cleavage. In addition, MHY-449-induced apoptosis proceeds via mitochondrial-mediated pathways involving activation and inhibition of Akt. These documented anticancer properties make MHY-449 an attractive candidate for further study in the prevention and therapy of lung cancer.

\section{Acknowledgements}

This study was supported by the National Research Foundation of Korea (NRF) grant, funded by the Korean Government (MSIP, no. 2009-0083538). We would like to thank the Aging Tissue Bank for providing research information.

\section{References}

1. Jung KW, Won YJ, Oh CM, Kong HJ, Cho H, Lee DH and Lee KH: Prediction of cancer incidence and mortality in Korea, 2015. Cancer Res Treat 47: 142-148, 2015.

2. Jemal A, Bray F, Center MM, Ferlay J, Ward E and Forman D: Global cancer statistics. CA Cancer J Clin 61: 69-90, 2011.

3. Jung KW, Won YJ, Kong HJ, Oh CM, Cho H, Lee DH and Lee KH: Cancer statistics in Korea: Incidence, mortality, survival, and prevalence in 2012. Cancer Res Treat 47: 127-141, 2015.

4. Govindan R, Page N, Morgensztern D, Read W, Tierney R, Vlahiotis A, Spitznagel EL and Piccirillo J: Changing epidemiology of small-cell lung cancer in the United States over the last 30 years: Analysis of the surveillance, epidemiologic, and end results database. J Clin Oncol 24: 4539-4544, 2006.

5. Kang JA, Yang Z, Lee JY, De U, Kim TH, Park JY, Lee HJ, Park YJ, Chun P, Kim HS, et al: Design, synthesis and anticancer activity of novel dihydrobenzofuro[4,5-b][1,8]naphthyridin-6-one derivatives. Bioorg Med Chem Lett 21: 5730-5734, 2011. 
6. Hwang HJ, Kang YJ, Hossain MA, Kim DH, Jang JY, Lee SH, Yoon JH, Moon HR, Kim HS, Chung HY, et al: Novel dihydrobenzofuro[4,5-b][1,8]naphthyridin-6-one derivative, MHY-449, induces apoptosis and cell cycle arrest in HCT116 human colon cancer cells. Int J Oncol 41: 2057-2064, 2012.

7. Lee SH, Kang YJ, Sung B, Kim DH, Lim HS, Kim HR, Kim SJ, Yoon JH, Moon HR, Chung HY, et al: MHY-449, a novel dihydrobenzofuro[4,5-b][1,8] naphthyridin-6-one derivative, induces apoptotic cell death through modulation of Akt/FoxOl and ERK signaling in PC3 human prostate cancer cells. Int J Oncol 44: 905-911, 2014.

8. Kroemer G and Reed JC: Mitochondrial control of cell death. Nat Med 6: 513-519, 2000.

9. Yamada T, Takeuchi S, Fujita N, Nakamura A, Wang W, Li Q, Oda M, Mitsudomi T, Yatabe Y, Sekido Y, et al: Akt kinase-interacting protein1, a novel therapeutic target for lung cancer with EGFR-activating and gatekeeper mutations. Oncogene 32: 4427-4435, 2013.

10. Zhou L, Luan H, Liu Q, Jiang T, Liang H, Dong X and Shang H: Activation of PI3K/Akt and ERK signaling pathways antagonized sinomenine-induced lung cancer cell apoptosis. Mol Med Rep 5: 1256-1260, 2012.

11. Lee MW, Kim DS, Lee JH, Lee BS, Lee SH, Jung HL, Sung KW, Kim HT, Yoo KH and Koo HH: Roles of AKT1 and AKT2 in non-small cell lung cancer cell survival, growth, and migration. Cancer Sci 102: 1822-1828, 2011.

12. Chandarlapaty S, Sawai A, Scaltriti M, Rodrik-Outmezguine V, Grbovic-Huezo O, Serra V, Majumder PK, Baselga J and Rosen N: AKT inhibition relieves feedback suppression of receptor tyrosine kinase expression and activity. Cancer Cell 19: 58-71, 2011.

13. Puglisi M, Thavasu P, Stewart A, de Bono JS, O'Brien ME, Popat S, Bhosle J and Banerji U: AKT inhibition synergistically enhances growth-inhibitory effects of gefitinib and increases apoptosis in non-small cell lung cancer cell lines. Lung Cancer 85: 141-146, 2014
14. Taylor RC, Cullen SP and Martin SJ: Apoptosis: Controlled demolition at the cellular level. Nat Rev Mol Cell Biol 9: 231-241, 2008.

15. Chang HY and Yang X: Proteases for cell suicide: Functions and regulation of caspases. Microbiol Mol Biol Rev 64: 821-846, 2000.

16. Tait SW and Green DR: Mitochondria and cell death: Outer membrane permeabilization and beyond. Nat Rev Mol Cell Biol 11: 621-632, 2010.

17. Kantari $\mathrm{C}$ and Walczak H: Caspase- 8 and bid: Caught in the act between death receptors and mitochondria. Biochim Biophys Acta 1813: 558-563, 2011.

18. Gross A, McDonnell JM and Korsmeyer SJ: BCL-2 family members and the mitochondria in apoptosis. Genes Dev 13: 1899-1911, 1999.

19. Stambolic V, Suzuki A, de la Pompa JL, Brothers GM, Mirtsos C, Sasaki T, Ruland J, Penninger JM, Siderovski DP and Mak TW: Negative regulation of PKB/Akt-dependent cell survival by the tumor suppressor PTEN. Cell 95: 29-39, 1998.

20. Kennedy SG, Wagner AJ, Conzen SD, Jordán J, Bellacosa A, Tsichlis PN and Hay N: The PI 3-kinase/Akt signaling pathway delivers an anti-apoptotic signal. Genes Dev 11: 701-713, 1997.

21. Kim D, Cheng GZ, Lindsley CW, Yang H and Cheng JQ: Targeting the phosphatidylinositol-3 kinase/Akt pathway for the treatment of cancer. Curr Opin Investig Drugs 6: 1250-1258, 2005.

22. Dinavahi SS, Prasanna R, Dharmarajan S, Perumal Y and Viswanadha S: A novel, potent, small molecule AKT inhibitor exhibits efficacy against lung cancer cells in vitro. Cancer Res Treat: Jan 2, 2015 (Epub ahead of print). doi: 10.4143/crt.2014.057.

23. Vasudevan KM, Gurumurthy S and Rangnekar VM: Suppression of PTEN expression by NF-kappa B prevents apoptosis. Mol Cell Biol 24: 1007-1021, 2004.

24. Jung IL, Kang HJ, Kim KC and Kim IG: PTEN/pAkt/p53 signaling pathway correlates with the radioresponse of non-small cell lung cancer. Int J Mol Med 25: 517-523, 2010. 